\title{
Serum heat shock protein 90 as a future predictive biomarker in childhood acute lymphoblastic leukemia
}

\author{
DOROTA PAWLIK-GWOZDECKA ${ }^{1}$, MAGDALENA GÓRSKA-PONIKOWSKA ${ }^{2}$, \\ ELŻBIETA ADAMKIEWICZ-DROŻYŃSKA ${ }^{l}$, MACIEJ NIEDŹWIECKI ${ }^{l}$
}

'Department of Pediatrics, Hematology, Oncology and Endocrinology, Medical University of Gdansk, Poland ${ }^{2}$ Department of Medical Chemistry, Medical University of Gdansk, Poland

\begin{abstract}
Heat shock proteins (HSPs) attract the attention of scientists and clinicians due to their potential role as diagnostic and prognostic factors in a variety of cancers. HSP90 is one of the most important and well-known family members, necessary for maintaining intracellular homeostasis. In the extracellular space, it is responsible for the transmission of alarm signals to the immune system. Numerous reports have indicated that the level of intra - and extracellular HSP90 can correlate either with a poorer prognosis or with a better outcome, depending on the type of cancer. Still, little is known why the level of this chaperone is increased in some tumors and decreased in others, reflecting dual role of protein in cell death processes. Currently, there is no database reporting levels of serum HSP9O in children with acute lymphoblastic leukemia (ALL). As such, using enzyme-linked immunosorbent assay (ELISA) method, we aimed to determine this parameter in a group of 21 patients with newly diagnosed ALL. We found decreased protein serum levels in patients at disease presentation and after induction block of chemotherapy in comparison to healthy controls. Furthermore, we observed a negative correlation between HSP90 serum levels and one of the earliest prognostic factors of the treatment responseperipheral blood lymphoblasts on the $8^{\text {th }}$ day of treatment. Our results indicate that HSP90 serum may play an important role in leukemogenesis and could be used as a marker to predict treatment failure in children with $A L L$.
\end{abstract}

Key words: acute lymphoblastic leukemia, cancer biomarkers, heat shock proteins (HSPS).

(Cent Eur J Immunol 2021; 46 (1): 63-67)

\section{Introduction}

Heat shock proteins (HSPs) are highly conserved proteins divided into several groups based on their molecular weights. One of them, HSP90, is a relatively well-known chaperone that has multiple functions, including regulation of cellular homeostasis. Under exposure to different kinds of environmental stressors, HSP90 expression increases in order to repair and stabilize damaged proteins such as oncoproteins involved in cancer cell growth. Upregulation of HSP90 is hypothesized to delay or prevent cells from entering the apoptotic pathway, which could account for the involvement of HSP90 in tumorigenesis and resistance to chemotherapy [1-3].

Initially, increased production of HSP90 was predominately associated with tumor aggressiveness and poorer prognosis [4]. A few studies confirmed the association between high expression levels of intracellular HSP90 and higher tumor staging in ovarian and gastric cancer pa- tients $[4,5]$. HSP90 upregulation is also associated with decreased survival in primary breast cancer [6].

However, nearly 50 years of observational data has revealed a more enigmatic nature of this protein because the intracellular concentration of HSP90 may, in certain cancers, correlate with an earlier cancer stage and an improved outcome [7].

The relatively recent discovery of extracellular HSPs has opened a possibility of using these as cancer biomarkers in clinical practice. An increased HSP90 in circulation was initially proposed to be an effect of cell death [8]. Since Hightower and Guidon reported that HSPs are also released by undamaged cells, the body of literature studying the mechanisms of HSP secretion into extracellular space has dramatically risen [9]. Several mechanisms have been proposed [10]. The prevailing view is that cytosolic HSPs are released via Golgi transport vesicles or via exosomes [11]. Serum HSP90, like other HSPs, was 
proposed to transmit signals to the immune system by interacting with $\mathrm{T}$ lymphocytes and stimulating natural killer (NK) cells. HSP90 also has immunomodulatory properties through increasing the expression of proinflammatory cytokines and stimulating the maturation of dendritic cells $[12,13]$. In the context of the anti-cancer response, HSP90 elicits cytotoxic reactions mediated by the innate and adaptive immune system. These facts support the hypothesis that circulating HSP90 elicits anti-cancer responses. However, this idea must be balanced by the pro-oncogenic role of HSP90 in promoting the invasion, angiogenesis, and metastasis of cancer [14]. In view of recent studies, the exact role of HSP90 in oncological diseases remains unclear.

Despite numerous well-known prognostic factors and good outcomes for patients who undergo chemotherapy treatment for acute lymphoblastic leukemia (ALL), the most common cancer in children, there are still patients with refractory leukemia or disease relapse. The most promising new therapies for ALL are based on immune system modulation using e.g. bi-specific monoclonal antibodies and over the past 5 years, these therapies have improved the prognosis of chemoresistant leukemic patients [15]. Also, autologous tumor-derived HSP peptide-based vaccines have demonstrated anti-tumor activity and excellent safety profiles in phase III clinical trials for advanced melanoma and renal cell carcinoma patients [16]. Therefore, searching for a novel prognostic and therapeutic agents is vital. Since there is no database reporting the level of serum HSP90 in children with ALL, we aimed to determine this parameter in a group of 21 newly diagnosed patients.

\section{Material and methods}

\section{Patients and samples}

The study was approved by the local research Ethics Committee. All patient samples and samples from normal controls were collected with a written informed consent previously obtained. Twenty-one children (10 male and 11 female), aged 2 to 14 years (median age, 4 years), with newly diagnosed acute lymphoblastic leukemia B-cell were enrolled into the study. Diagnosis was performed using cytological, cytochemical, and immunophenotyping methods. In the study group, blood samples were collected twice: before chemotherapy and at the day of evaluation of remission status ( $33^{\text {rd }}$ day of therapy). Between these two time points, all patients received an intensive block of chemotherapy - according to IA of Berlin-Frankfurt-München protocol, with an induction using prednisone $60 \mathrm{mg} / \mathrm{m}^{2} /$ day for 33 days, vincristine $1.5 \mathrm{mg} / \mathrm{m}^{2} /$ day, daunorubicin $30 \mathrm{mg} / \mathrm{m}^{2} /$ day, 4 times during the protocol, and L-asparaginase $5000 \mathrm{U} / \mathrm{m}^{2} /$ day, 8 times. One patient had a BCR/ABL mutation, therefore according to standard treatment, imatinib was applied. As a result, at the end of the protocol,
15 of 21 patients were classified into intermediate-risk (IR) group, 5 into high-risk (HR) group, and only one patient was categorized into standard-risk (SR) group.

\section{Evaluation of HSP90 concentration}

Obtained blood samples were allowed to clot at room temperature for $1 \mathrm{~h}$, centrifuged at $1,000 \times \mathrm{g}$ for $20 \mathrm{~min}$, and stored at $-80^{\circ} \mathrm{C}$ for later analysis. Serum HSP90 concentrations were evaluated by enzyme-linked immunosorbent assay (ELISA) according to the manufacturer's instruction (human serum HSP90 ELISA kit, Cloud-Clone Corp., Houston, USA). Samples and standards were added and incubated for $1 \mathrm{~h}$. The conjugate antibody was then added and incubated for additional $30 \mathrm{~min}$. Plates were washed, and the substrate was added to develop the color change and incubated for $20 \mathrm{~min}$ at $37^{\circ} \mathrm{C}$. Finally, optical densities were read at $450 \mathrm{~nm}$ and the samples were compared to the standards. HSP levels are presented in units of $\mathrm{ng} / \mathrm{ml}$.

\section{Statistical analysis}

Statistical analysis was performed using Statistica Software version 7.1 for Windows (StatSoft Inc., 2005). Mann-Whitney tests were used to compare the differences between the normal control and leukemic patient, and Wilcoxon test was used to indicate alterations in HSP90 serum level in the research group before and after chemotherapy. The correlation between prognostic factors and serum concentration of HSP90 in leukemic patients were analyzed using Spearman's correlation analysis. $P<0.05$ was considered as statistically significant.

\section{Results}

We investigated serum HSP90 levels in a group of 21 pediatric patients with acute lymphoblastic leukemia. The median serum HSP90 level on the day of the diagnosis was $3.45 \mathrm{ng} / \mathrm{ml}$ (range, $1.22-23.85 \mathrm{ng} / \mathrm{ml}$ ) and on the $33^{\text {rd }}$ day of therapy, it was $4.79 \mathrm{ng} / \mathrm{ml}$ (range, $1.07-52.51 \mathrm{ng} / \mathrm{ml}$ ). Median serum level of HSP90 in controls was $12.43 \mathrm{ng} / \mathrm{ml}$ (range, 3.16-33.58 ng/ml). We found statistical differences between the serum level of HSP90 in ALL patients between two time points (Fig. 1; $\mathrm{Z}=-2.07 ; p=0.039 ; \eta^{2}=0.13$, Wilcoxon signed-rank test). $\eta^{2}$ revealed a medium-strength effect. Patients at disease presentation and on $33^{\text {rd }}$ day of therapy showed decreased serum in HSP90 levels compared to healthy controls (Fig. 2; baseline: $\mathrm{U}=41, p=0.001$, $\eta^{2}=0.33$, and $33^{\text {rd }}$ day: $U=82, p=0.023, \eta^{2}=0.15$, Mann-Whitney test). Both effects were strong, but the difference between patients and controls was higher on the day of diagnosis than after $33^{\text {rd }}$ day ( $\eta^{2}$ was bigger more than twice).

Next, we evaluated the association of serum HSP90 with a series of clinical and hematological agents. Due to the insufficient number of patients, we were unable to 


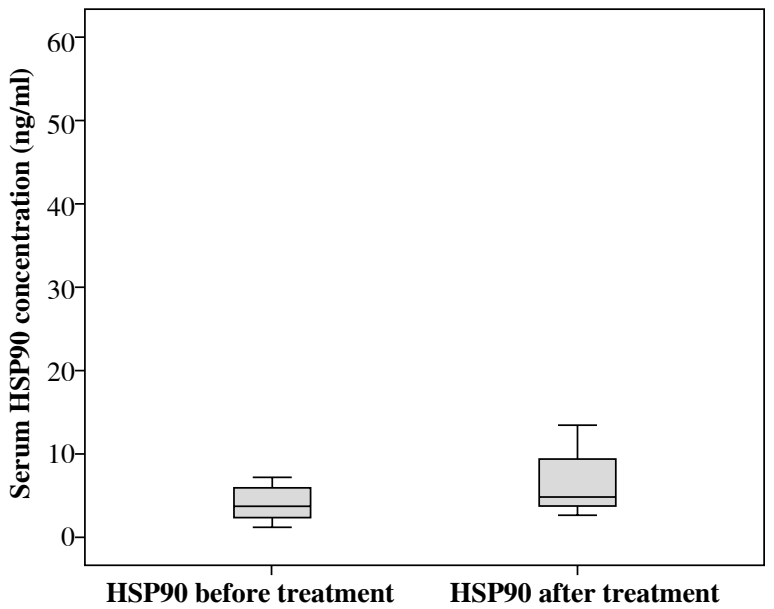

Fig. 1. HSP90 serum level before and after induction chemotherapy

analyze cytogenetic, immunophenotypic factors, and central nervous system (CNS) status in the context of serum HSP90. We found no association between the concentration of HSP90 in circulation and age, gender, weight, or height. Further, there was no association between HSP90 serum levels and WBC levels, bone marrow, and peripheral blood blast percentages at presentation and minimal residual disease (MRD), or peripheral blood and bone marrow blasts percentages during treatment. Interestingly, we observed non-significant negative correlation between serum HSP90 and lymphoblasts on the $8^{\text {th }}$ day of chemotherapy $\left(r_{\mathrm{s}}=-0,34 ; p=0.192 ;\right.$ Fig. 3$)$.

\section{Discussion}

HSPs are highly conserved molecules presented in both prokaryotic and eukaryotic cells [17]. They regulate a variety of critical cellular processes and repair misfolded proteins, including oncoproteins, and therefore have been studied in the context of carcinogenesis [3]. Despite years of continued investigation on the role of HSPs in the tumor biology, little is known about the clinical and laboratory association between the intra- and extracellular levels of HSP90 in acute lymphoblastic leukemia in children. Indeed, there was only one report on this parameter in pediatric ALL patients. There are also a limited number of reports concerning HSPs in adult acute lymphoblastic leukemia due to rarity of the disease in patients over 18 years old.

The aim of our study was to evaluate serum levels of HSP90 in a pediatric group of ALL patients before and after induction block of chemotherapy. We also investigated the potential diagnostic and prognostic impact of the protein.

We demonstrated that the serum concentration of HSP90 on the day of the diagnosis was lower when compared to controls (Fig. 2). This is an interesting finding,

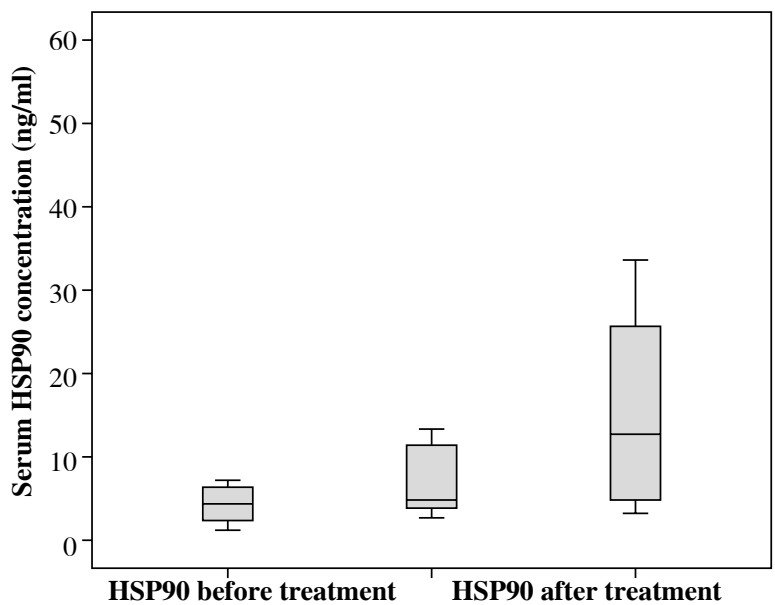

Fig. 2. Decreased serum HSP90 levels of patients at disease presentation and after induction chemotherapy compared to healthy controls

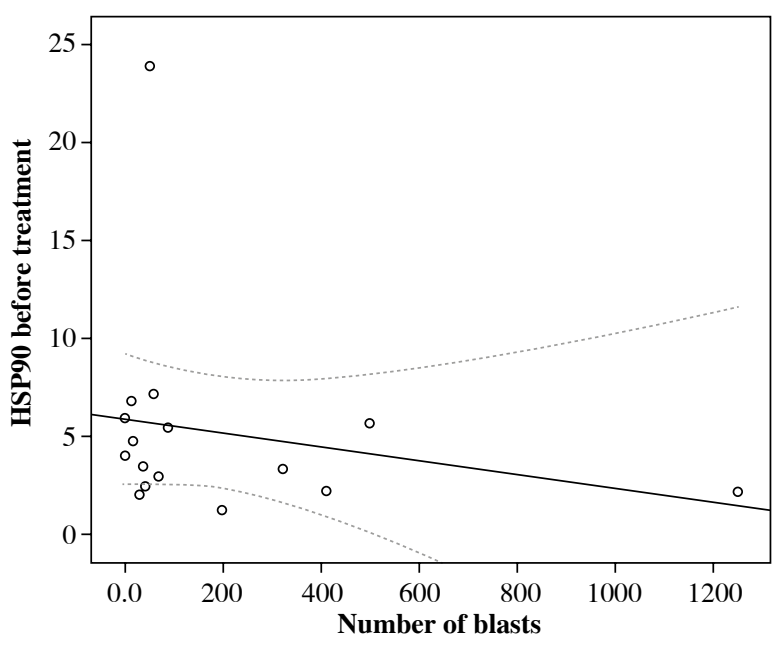

Fig. 3. Correlation between serum HSP90 and lymphoblasts on the $8^{\text {th }}$ day of chemotherapy

since previous studies conducted in adults reported significantly higher levels of the protein at presentation [18]. Increased levels of HSP90 serum was also observed in a study conducted on animals. The researchers reported that mice transplanted with adult B-cell leukemia showed higher extracellular HSP90 levels in comparison to healthy mice. This fact confirms that circulating HSP90 is not only the consequence of cancer cells necrosis, but the level is the matter of complicated mechanisms of regulation. This also suggests major differences in molecular pathomechanisms between childhood and adult acute leukemia [19]. In our opinion, these differences are due to distinct patterns of cancer response caused by "uneducated" adaptive immune system of children (ALL in children arise from naive 
B cells at pre-antigen stages, while ALL in adults is frequently manifested by post-antigen stimulated cells) [20].

We hypothesize that decreased level of circulating HSP90 can be an expression of unknown mechanisms that maintain an immune tolerance, enabling the development of leukemia. Further studies are crucial to determine the cause of differences.

In the literature, we found only one study reporting on protein expression level in ALL. The authors showed up-regulation of HSP90 gene in leukemia cells derived from bone marrow of pediatric patients compared to peripheral blood mononuclear cells (PBMC) of healthy controls. The higher HSP90 expression was explained by an increased cell differentiation and proliferation. However, a limitation of the study was a comparison of cells obtained from bone marrow of ALL patients with PMBCs of healthy controls [21]. We are the first, to our knowledge, who show that the serum level is not parallel to HSP90 gene expression in bone marrow. However, further study evaluating the two parameters is needed.

Additionally, we investigated the response of serum HSP90 level in chemotherapy induction and found that the concentration of the protein in circulation increased in most of the patients when compared to levels before chemotherapy (Fig. 1). Therefore, we were able to observe that the serum concentration of HSP90 was not stable during treatment. The reason for this, in our opinion, was the impact of cytostatics on the expression or the secretion of the molecule rather than reduced number of cancer cells. This hypothesis is supported by Venkatakrishnan et al., who found altered expression levels of HSP27 after doxorubicin therapy [22]. We found no correlations between HSP90 levels at presentation and after induction chemotherapy with the percentage of bone marrow blasts, which reflect the total tumor load. However, an estimation of leukemia cell burden, especially at presentation, is not accurate due to organ infiltration of these cell.

In the present study, we also evaluated serum HSP90 in the context of clinical and laboratory factors. No relationship between the parameter and sex, age, white blood counts, lymphoblasts, and the bone marrow lymphoblast percentage at presentation as well as MRD and bone marrow blasts percentage during treatment were observed. Interestingly, we found a negative correlation between the concentration of serum HSP90 before treatment and the percentage of lymphoblasts in peripheral blood on the $8^{\text {th }}$ day of therapy (Fig. 3), which is one of earliest prognostic factors of treatment response and reflects sensitivity of cancer cells to steroids. The glucocorticoid receptor, which is responsible for the intracellular effects of the drug, is bound in its inactive form in a multiprotein complex with HSP90. Lack of HSP90 was demonstrated to be related to glucocorticoid resistance in human lesion-derived cell lines [23]. Also, the expression of aberrant HSP90 protein was associated with glucocorticoid resistance in leukemic cell lines [24]. Our results are consistent with these studies. Therefore, we suppose that extracellular HSP90 can be an early predictive biomarker of the response of cancer cells to therapy in childhood ALL. However, Lauten et al. did not find a relationship between HSP90 expression level in bone marrow and resistance to steroids in children with ALL. Further studies are needed to better understand the role of serum HSP90 in the prognosis of ALL treatment response [25].

\section{Conclusions}

To our knowledge, we are the first to report a decreased HSP90 serum level in childhood ALL. Moreover, we found that low concentration of HSP90 correlated with poor response to steroids in children with ALL. However, small number of patients was a significant limitation to the study. Additional studies with a larger patient sampling size are needed to determine the clinical significance of extracellular HSPs in the pathogenesis of ALL, and whether they can be used for the diagnosis and prognosis of cancer.

\section{Acknowledgments}

This study was supported by a grant for young researchers (MN 01-0262/08/152) from the Medical University of Gdańsk, Poland.

\section{The authors declare no conflict of interest.}

\section{References}

1. Seigneuric R, Mjahed H, Gobbo J, et al. (2011): Heat shock proteins as danger signals for cancer detection. Front Oncol 1: 37 .

2. Darimont BD (1999): The Hsp90 chaperone complex A potential target for cancer therapy. World J Gastroenterol 5: 195-198.

3. Calderwood SK (2018): Heat shock proteins and cancer: intracellular chaperones or extracellular signalling ligands? Philos Trans R Soc Lond B Biol Sci 373: 20160524.

4. Wang J, Cui S, Zhang X, et al. (2013): High expression of heat shock protein 90 is associated with tumor aggressiveness and poor prognosis in patients with advanced gastric cancer. PLoS One 8: e62876.

5. Elpek GO, Karaveli S, Simsek T, et al. (2003): Expression of heat-shock proteins Hsp27, Hsp70 and Hsp90 in malignant epithelial tumor of the ovaries. APMIS 111: 523-530.

6. Pick E, Kluger Y, Giltnane JM, et al. (2007): High HSP90 expression is associated with decreased survival in breast cancer. Cancer Res 67: 2932-2937.

7. Ciocca DR, Calderwood SK (2005): Heat shock proteins in cancer: diagnostic, prognostic, predictive, and treatment implications. Cell Stress Chaperones 10: 86-103.

8. Basu S, Binder RJ, Suto R, et al. (2000): Necrotic but not apoptotic cell death releases heat shock proteins, which deliver a partial maturation signal to dendritic cells and activate the NF- $\kappa$ B pathway. Int Immunol 12: 1539-1546. 
9. Hightower LE, Guidon PT (1989): Selective release from cultured mammalian cells of heat-shock (stress) proteins that resemble glia-axon transfer proteins. J Cell Physiol 38: 257266.

10. Didelot C, Lanneau D, Brunet M, et al. (2007): Anti-cancer therapeutic approaches based on intracellular and extracellular heat shock proteins. Curr Med Chem 14: 2839-2847.

11. Campanella C, Caruso Bavisotto C, Marino Gammazza A, et al. (2014): Exosomal heat shock proteins as new players in tumour cell-to-cell communication. J Circ Biomark 3: 5.

12. Binder RJ (2014): Functions of heat shock proteins in pathways of the innate and adaptive immune system. J Immunol 193: 5765-5771.

13. Bae J, Munshi A, Li C, et al. (2013): Heat shock protein 90 is critical for regulation of phenotype and functional activity of human T lymphocytes and NK cells. J Immunol 190: 1360-1371.

14. Santos TG, Martins VR, Hajj GNM (2017): Unconventional secretion of heat shock proteins in cancer. Int J Mol Sci 18: 946.

15. Le Jeune C, Thomas X (2016): Potential for bispecific T-cell engagers: role of blinatumomab in acute lymphoblastic leukemia. Drug Des Dev Ther 10: 757-765.

16. Eton O, Ross MI, East MJ, et al. (2010): Autologous tumor-derived heat-shock protein peptide complex-96 (HSPPC-96) in patients with metastatic melanoma. J Transl Med 8: 9.

17. Lee JW, Cho B (2017): Prognostic factors and treatment of paediatric acute lymphoblastic leukaemia. Korean J Pediatr 60: 129-137.

18. Ma XJ, Zhang FH, Sun L (2017): Expression and Significance of PD-L1, HSP90 and HSP90 $\alpha$ in Serum of Patients with Acute Leukemia. Zhongguo Shi Yan Xue Ye Xue Za Zhi 25: 1384-1389.

19. Milani M, Laranjeira ABA, de Vasconcellos JF, et al. (2015): Plasma Hsp90 Level as a Marker of Early Acute Lymphoblastic Leukemia Engraftment and Progression in Mice. PLoS One 10: e0129298.

20. Whitehead TP, Metayer C, Wiemels JL, et al. (2016): Childhood leukemia and primary prevention. Curr Probl Pediatr Adolesc Health Care 46: 317-352.

21. Yufu Y, Nishimura J, Nawata H (1992): High constitutive expression of heat shock protein $90 \alpha$ in human acute leukemia cells. Leuk Res 16: 597-605.

22. Venkatakrishnan CD, Tewari AK, Moldovan L, et al. (2006): Heat shock protects cardiac cells from doxorubicin-induced toxicity by activating p38MAPK and phosphorylation of small heat shock protein 27. Am J Physiol Heart Circ Physiol 29: H2680-H2691.

23. Bray PJ, Du B, Mejia VM, et al. (1999): Glucocorticoid resistance caused by reduced expression of the glucocorticoid receptor in cells from human vascular lesions. Arterioscler Thromb Vasc Biol 19: 1180-1189.

24. Kojika S, Sugita K, Inukai T, et al. (1996): Mechanisms of glucocorticoid resistance in human leukemic cells: implication of abnormal 90 and $70 \mathrm{kDa}$ heat shock proteins. Leukemia 10: 994-999.

25. Lauten M, Beger C, Gerdes K, et al. (2003): Expression of heat-shock protein 90 in glucocorticoid-sensitive and -resistant childhood acute lymphoblastic leukaemia. Leukemia 17: 1551-1556. 